\title{
Depth Dependence Investigation of Manganese Charge State in Oxygen-deficient LSMO Thin Films
}

\author{
Aubrey N. Penn ${ }^{1}$, Abinash Kumar ${ }^{1}$, Robbyn Trappen ${ }^{2}$, Guerau Cabrera ${ }^{2}$, Mikel B. Holcomb ${ }^{2}$, and James \\ M. LeBeau ${ }^{1}$ \\ 1. Department of Materials Science and Engineering, North Carolina State University, Raleigh, NC USA \\ 2. Department of Physics and Astronomy, West Virginia University, Morgantown, WV USA
}

Lanthanum strontium manganite (LSMO) complex oxide thin films are of interest for applications with colossal magnetoresistance, half-metallic behavior, high conductivity, and room temperature ferromagnetism. Additionally, LSMO thin films exhibit charge redistribution when interfaced with other complex oxides such as strontium titanate $[1,2]$. A major limiting factor of these metal oxides, however, is that they lose magnetic behavior and metallicity in exceedingly thin films due to electronic and structural reconstructions [3]. Near the interfaces, for example, the $\mathrm{MnO}_{6}$ octahedra in these perovskites are found to distort. Furthermore, a mixture of $\mathrm{Mn}^{2+}, 3+$ and 4+ charge states exist in LSMO, and it is widely observed that the oxidation state decreases exponentially as the interface is approached [4]. Because dead layers and $\mathrm{Mn}$ valence reduction both occur within a several unit cell range, extracting spatially resolved electronic and structural information is of utmost importance for identifying mechanisms leading to degraded properties.

In this presentation, we will report on the variation of $\mathrm{Mn}$ charge state and local crystallographic distortion as a function of oxygen deficiency. The $\mathrm{La}_{0.33} \mathrm{Sr}_{0.67} \mathrm{MnO}_{3}$ films, grown on $\mathrm{SrTiO}_{3}$ by pulsed laser deposition [2], are found to require careful sample preparation, particularly during $\mathrm{Ar}^{+}$ion milling [6] to capture the true electronic structure of the interface. To investigate local strain, we will present results from Revolving STEM (RevSTEM), to determine differences of the interface structure and to quantify octahedral distortion [7]. For example, as shown in Figure 1(b), structural differences arise at the LSMO/STO interface in the oxygen deficient sample as compared to the stoichiometric interface in Figure 1(a). A systematic reduction of intensity is present along the interface, indicative of point defect segregation and corresponding lattice disorder. As shown in Figure 1(c), the atom column near neighbor distances increase.

To determine Mn charge state variation, electron energy loss spectroscopy results will also be discussed. For instance, the $\mathrm{L}_{3} / \mathrm{L}_{2}$ ratio acquired from EELS is shown in Figure 2. Note that the ratio and charge state are inversely proportional, therefore increased ratio at the interface indicates a decreasing Mn charge state [1]. In contrast, the stoichiometric sample does not exhibit this dramatic interfacial change in valence. This trend is explained by the breakdown of the double-exchange mechanism in oxygen vacant systems, inducing electron doping at the interface [3]. Finally, we will discuss how these results can explain features in the magnetic measurements as a function of oxygen content during growth. 


\section{References:}

[1] J. Mundy, et al., Nature Communications 5 (2014), p. 1-6.

[2] J. Zhou, et al., Appl. Phys. Lett. 107 (2015), p. 141603.

[3] R. Peng, et al., Appl. Phys. Lett. 104 (2014), p. 081606.

[4] Y. Moritomo, et al., Nature 380 (1996), p. 141-144.

[5] H. Tan, et al., Ultramicroscopy 116 (2012), p. 24-33.

[6] L. Dieterle, et al., Ultramicroscopy 111 (2011) p. 1636-1644.

[7] X. Sang, et al., Ultramicroscopy 138 (2014) p. 28-35.

[8] This work is supported by the National Science Foundation (DMR-1608656). The authors also acknowledge the Analytical Instrumentation Facility (AIF) at North Carolina State University, which is supported by the State of North Carolina and the National Science Foundation.
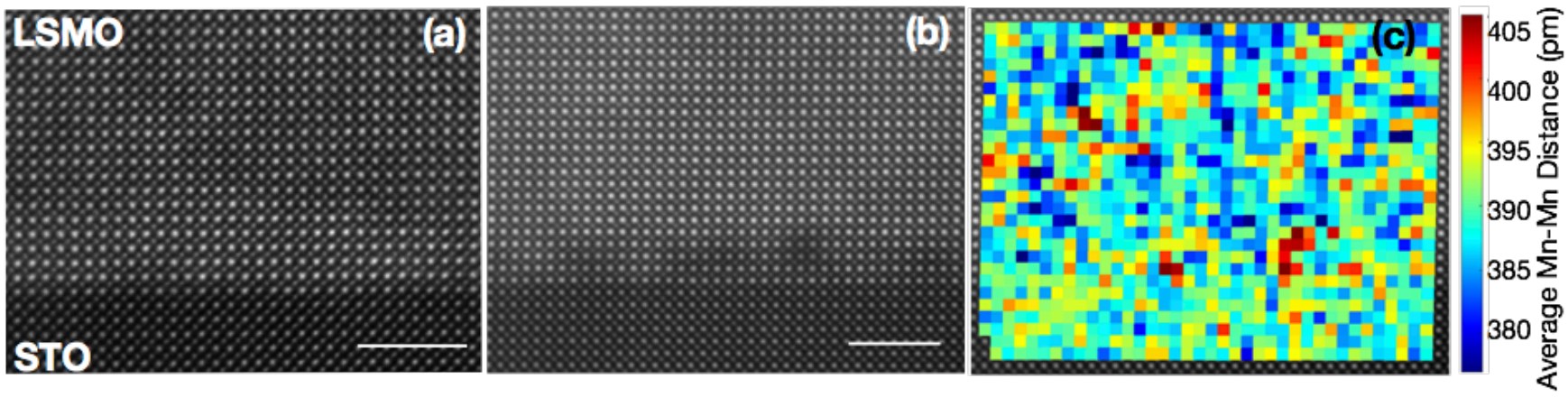

Figure 1. RevSTEM images of stoichiometric (a) and oxygen deficient (b) LSMO thin films. (c) MnMn displacement of oxygen deficient LSMO, showing increased spacing in regions with defects. (Scale bar - $3 \mathrm{~nm})$.

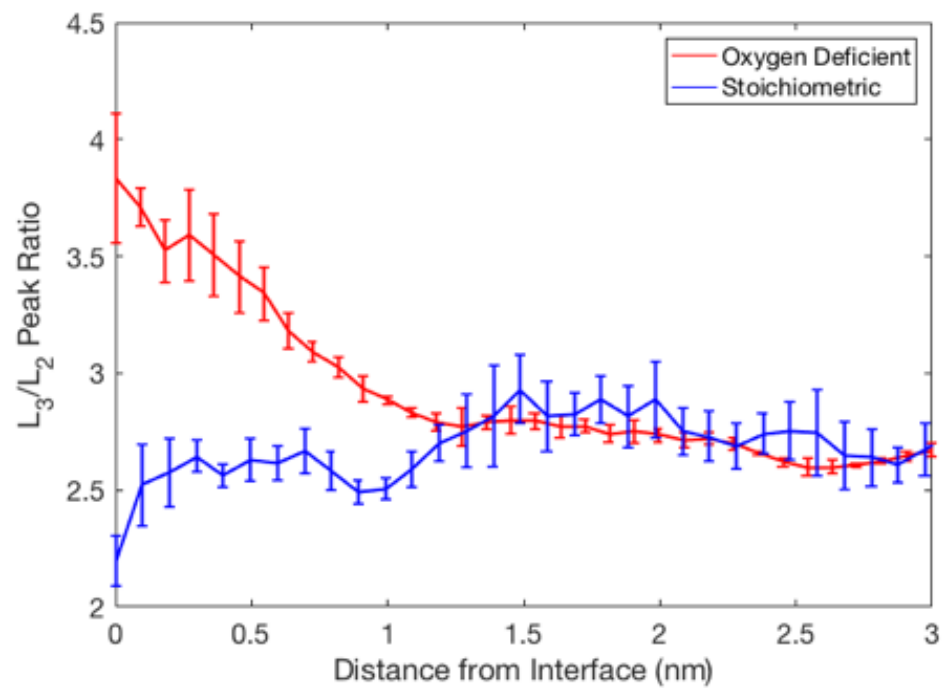

Figure 2. $\mathrm{L}_{3} / \mathrm{L}_{2}$ white line integrated peak ratio of oxygen deficient and stoichiometric LSMO. The change in Mn charge is inversely correlated to this ratio, which can be correlated with low temperature magnetic measurements conducted on the same films. 